\title{
Evaluating the Impact of Job Training and Housing Assistance Programs on the Dynamics of Homelessness Los Angeles County of California: A Mathematical Modeling Study
}

\author{
Asma Azizi ${ }^{\mathrm{a}}$, Jackson Carpenter ${ }^{\mathrm{b}}$, Mohammad Alharbi ${ }^{\mathrm{c}, \mathrm{d}}$, Jeniffer Sánchez ${ }^{\mathrm{e}}$, \\ Vincent Jones $^{\mathrm{f}}$, Cesar Montalvo ${ }^{\mathrm{a}}$, Viswanathan Arunachalam ${ }^{\mathrm{g}}$, Anuj Mubayi ${ }^{\mathrm{a}}$ \\ ${ }^{a}$ Simon A. Levin Mathematical, Computational and Modeling Sciences Center, Arizona \\ State University, Tempe, Arizona, U.S.A \\ ${ }^{b}$ Department of Mathematics, Arizona State University, Tempe, Arizona, U.S.A \\ ${ }^{c}$ Department of Mathematics, University of Texas at Arlington, Arlington, Texas, U.S.A \\ ${ }^{d}$ Department of Mathematics, University of Jeddah, Jeddah, Makkah, Saudi Arabia \\ ${ }^{e}$ Department of Physics, Yachay Tech University, San Miguel de Urcuqui Canton, Imbabura \\ ,Ecuador \\ ${ }^{f}$ Department Department of Mathematics, University of Pennsylvania, Philadelphia, U.S.A \\ ${ }^{g}$ Departamento de Estadística Facultad de Ciencias Universidad Nacional de Colombia, \\ Bogotá, Colombia
}

\begin{abstract}
Homelessness is a major socioeconomic issue in the United States, with second highest rate of homelessness in Los Angeles (L.A.) County with around 53, 000 individuals, $10 \%$ of the national total homelessness. In the literature, homelessness has been shown to be associated with various factors including poor family functioning, socioeconomic disparity, and isolation from social network communities. Recent interventions such as rapid rehousing, and job training have either been ineffective or have had limited scope over time. In this study, we use a dynamical mathematical model to understand dynamics of homelessness and to evaluate the impact of two intervention programs on its control in L.A., California. The model assumes that the homelessness is spread in a population via social and environment influences and regulated by housing assistance and job training interventions. Our analysis suggests that temporary housing assistance program is more effective in reducing homelessness in a short run, however, to achieve sustainable long lasting low levels in homelessness, job training program needs to be focused.
\end{abstract}

Keywords: Homelessness, Job training, Housing assistance, Compartmental ODE model, Levine's model, Sensitivity Analysis

Email address: amubayi@asu.edu (Anuj Mubayi) 


\section{Introduction}

Homelessness is defined-by the Department of Housing and Urban Development (HUD) - as a lack of a fixed, regular, and adequate nighttime residence. Vulnerable individuals to homelessness suffer from a variety of factors, including poverty [1], lack of network support [2, 3, 4, 5, poor health, mental and physical disabilities [1, 6, and unemployment [8, 7, 9. Moreover, socioeconomic factors, such as living in impoverished communities (having an income less than $\$ 15,000$ per individual per year [10]), plays fundamental roles in the homelessness process via generating a cycle of poverty, resulting in a homeless person's inability to afford housing or rent.

Although the total number of homeless people in the United States has dropped by $15 \%$ during 2007 - 2017, there was a slight increment of $0.7 \%$ in the homelessness rate over the year of 2018 [11]. Los Angeles, California, (L.A.) is one of the most populous counties in the United States with a highest rate of homelessness, US Interagency Council on Homelessness (USICH). According to HUD , 53,000 L.A. County residents were homeless on any given night in 2018. In 2018, about $15 \%$ of L.A. County residents lived under the poverty line that spend $71 \%$ of their income on housing [12, in contrast to $12.3 \%$ across the entire United States [13. The lack of low-cost housing and high poverty rates may be some of the leading factors for high rate of homelessness in L.A [14.

The strategies to control homelessness are, but not limited to, job training and housing assistance. To reduce the risk of becoming homeless, job training scheme was offered by The Roberts Enterprise Development Fund (REDF) program for unemployed population [15]. Within this program, participants are trained to build soft, vocational, or technical skills along with developing basic skills through regular classes to rejoin the workforce. On the other hand, housing assistance is a temporary housing strategy for homeless individuals given by the Continuum of Care $(\mathrm{CoC})$ program [16]. This program is provided by state and local governments to quickly and temporarily rehouse homeless individuals and families while minimizing the trauma and dislocation costs to homeless individuals [16.

In spite of the need for rigorous mechanistic models to understand the dynamics of homelessness and to provide more insight on the effectiveness of current interventions (hosing assistance and job training), a wide range of works focus on data analysis and statistical model with a little or no effort on evaluating these interventions $11,17,18,19,20,21,22$. We only found three very simplistic mathematical models related to this social challenge, [23, 24, 25]. Wodon et al. 23] used an optimal control model to compare two scenarios of hosing assistance: providing housing assistance for low income population or shelter for homeless individuals. They found out though it is less costly, providing shelter for homeless individuals does not have long-term impact on homelessness alleviation as housing assistance for low income people has. A linear compartmental model was developed by Lacey et al. 24 to understand the impact of council housing on the control of homelessness in the UK. Through simulation they observed that an increment in the transition of homeless individuals into 
council stock caused a dramatic increase in the homeless population. However, reducing this transition has little effect on the sizes of other categories on the waiting list. Using a mathematical model, Bhunu et al. [25] coupled the spread of homelessness and drug-misuse to understand how they effect one another. Because of the homelessness and drug-misuse positively enhances each other, it is required to effectively control these two social problems simultaneously in order to reduce homelessness [25].

The focus of our study is to evaluate the impact of housing assistance and job training programs on the spread and or alleviate homelessness in L.A., especially under the presence of disparities in socioeconomic conditions related to

a neighborhood. In particular we develop and analyze a compartmental model with a deterministic and stochastic system of differential equations in which homelessness is not only conceptualized from socioeconomic perspective, but also from the ecological perspective as the product of the dynamic interplay between individuals and their environments 26, 27. The model is parametrized by linking it with data from LA county and performing parameter sensitivity analysis on relevant model outputs. The results not only quantified the variation in homelessness but also evaluated dynamics of it as a function of environmental and social factors. Furthermore, this study also attempts to understand the efficiency of two proposed interventions.

This paper is laid out as follows: First, the model's development is described along with assumptions and parameters explanation. Then, the mathematical analysis is detailed with the model to highlight the effect of the different interventions in the system. The Numerical Section presents numerical results, including parameter estimation, numerical simulations, and sensitivity analysis. Finally, Conclusion Section presents the main results of the study.

\section{Method}

\subsection{Data source}

We collect the time-series data on the number of people under poverty line, the number of people under poverty line who lost their job, and the number of homeless people reside in California for the past 10 years [12, 34, 35, 36. We also collect the data on poverty rate in California on the same time interval [12, 34, 35, 36. This data will be used to estimate the unknown parameters of the model explained in detail in the following subsection.

\subsection{The deterministic model}

We define Vulnerable (V) as residents of L.A. County ages 18 or more and live under the poverty line, that is, the their income is less than $\$ 15,000$ per year per individual [10, or they are living near a disaster area 29, 28. The Disaffiliated (D) class is comprised of those individuals from the vulnerable class that have lost their job. Grigsby et al. defined disaffiliation as a process of increasing detachment from traditional institutions and social roles [7. Thus, in the case of this study, unemployment is one form of disaffiliation from the traditional 
workforce. Disaffiliated individuals are more likely to become Homeless (H) through coping with their distress by re-affiliating with homeless individuals [7]. Disaffiliated individuals can be influenced by the environment as well [26, 27. A similar approach was presented by Levins et al., Levins model 30. Their model describes the time-dependent changes in the population of a species in a certain area and how it varies depending on the extinction and colonization of sub-populations [31. In our model, the environmental influence is represented by the fraction of the city in which people live in high poverty stages, which is defined as a compartment representing an indirect influence affecting the transition from a disaffiliated to a homeless state. Colonization and extinction rates would be represented by the increment or reduction of the high poverty areas, respectively.

The Levin's model is an example of patch occupancy meta-population model, which in our case are associated with high poverty community areas. In order to adopt the Levin's model, we consider the following assumptions 32 .

1. The suitable habitat occurs in infinitely many patches that are equally large and of the same quality.

2. The patches have only two possible states, occupied or empty, the area is occupied by a high poverty community, not considering higher socioeconomic levels.

3. Local extinctions and colonization occur independently in different patches, and

4. all local populations are equally connected to other populations and patches.

Based on these assumptions, we define the state variables and parameters of the proposed model in the Table 1. In the model, we introduce two intervention strategies: job training implemented on disaffiliated population and housing assistance with the focus on providing temporary shelter for homeless population. Disaffiliated and homeless individuals have their own rates of entering and leaving their respective programs. These assumptions and definitions result in the following system of nonlinear differential equations:

$$
\left\{\begin{array}{l}
\frac{d V}{d t}=\mu N-\sigma V-\mu V+\kappa_{D} s F_{D} \\
\frac{d D}{d t}=\sigma V-\beta(H, p) D-\mu D-\gamma_{D} D+\kappa_{H} q F_{H}+k_{D}(1-s) F_{D} \\
\frac{d H}{d t}=\beta(H, p) D-\mu H-\gamma_{H} H+\kappa_{H}(1-q) F_{H} \\
\frac{d F_{H}}{d t}=\gamma_{H} H-\kappa_{H} F_{H}-\mu F_{H} \\
\frac{d F_{D}}{d t}=\gamma_{D} D-\kappa_{D} F_{D}-\mu F_{D} \\
\frac{d p}{d t}=\Omega(H) p(1-p)-\varepsilon p
\end{array}\right.
$$

where, $N=V+D+H+F_{H}+F_{D}$, is the total conserved population, $\mu$ is per capita death rate, $\sigma$ is the rate of losing job for vulnerable individuals. The parameter $\beta(H, p)$ is the nonlinear transition rate from disaffiliated class to 
homeless class. We define $\beta(H, p):=\beta_{p} p+\beta_{H} \frac{H}{N}$. The $\beta_{p} p$ term incorporates the influence of high poverty communities, whereas $\beta_{H} \frac{H}{N}$ incorporates the influence of homeless on disaffiliated individuals. The term $\Omega(H)(1-p)$ is the nonlinear colonization rate with $\Omega(H):=\frac{H}{N}$. For simplicity, this linear definition of $\Omega(H)$ assumes that as the homeless population of L.A. increases, so do the high poverty communities of L.A. County. The parameter $\epsilon$ stands for the rate of emigration out of high poverty area, and is called poverty alleviation rate. The Figure (1) shows the schematic diagram and the Table (1) lists the definition for the variables and parameters of our model (1).

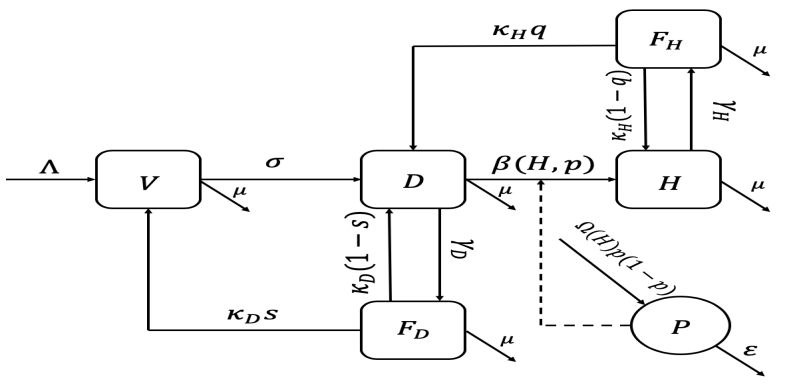

Figure 1: Schematic of the model

\begin{tabular}{|c|c|c|}
\hline \multirow{7}{*}{$\begin{array}{l}\text { State } \\
\text { variables }\end{array}$} & Notation & Definition \\
\hline & $V(t)$ & Vulnerable individuals at time $t$ (Person) \\
\hline & $D(t)$ & Disaffiliated individuals at time $t$ (Person) \\
\hline & $H(t)$ & Homeless individuals at time $t$ (Person) \\
\hline & $F_{D}(t)$ & Individuals under job training at time $t$ (Person) \\
\hline & $F_{H}(t)$ & Individuals under temporary housing at time $t$ (Person) \\
\hline & $p(t)$ & Fraction of environment under high poverty (1) \\
\hline \multirow{11}{*}{ Parameters } & $\mu$ & Per capita death rate $(1 /$ Time $)$ \\
\hline & $\sigma$ & Job losing rate $(1 /$ Time $)$ \\
\hline & $\beta_{p}$ & $\begin{array}{l}\text { Environment influence rate, related to neighborhood } \\
\text { poverty status }(1 / \text { Time })\end{array}$ \\
\hline & $\beta_{H}$ & $\begin{array}{l}\text { Homeless influence rate, which is direct social influence rate } \\
\text { between homeless and disaffiliated individuals (1/Time) }\end{array}$ \\
\hline & $\gamma_{D}$ & job training rate $(1 /$ Time $)$ \\
\hline & $\gamma_{H}$ & Housing assistance rate $(1 /$ Time $)$ \\
\hline & $\kappa_{D}$ & Rate of leaving job training (1/Time) \\
\hline & $\kappa_{H}$ & Rate of leaving housing assistance (1/Time) \\
\hline & $s$ & $\begin{array}{l}\text { Job training success: fraction of individuals obtain job after } \\
\text { job training (1) }\end{array}$ \\
\hline & $q$ & $\begin{array}{l}\text { Housing assistance success: fraction of individuals obtain } \\
\text { permanent house after a housing assistance (1) }\end{array}$ \\
\hline & $\epsilon$ & poverty alleviation rate $(1 /$ Time $)$ \\
\hline
\end{tabular}

Table 1: State variable and parameters definition and their units. 


\subsection{The stochastic model}

Beside the deterministic model, we complete stochastic modelling with a Continuous Time Markov Chain process (CTMC) and the Gillespie algorithm. Defining the vector $X(t)=\left[V(t), D(t), H(t), F_{H}(t), F_{D}(t), p(t)\right]$, we give the probabilities of 16 events with the possible state change at the probabilities shown in Table (2).

\begin{tabular}{clll}
\hline $\boldsymbol{\Delta} \boldsymbol{X}$ & Probability & $\boldsymbol{\Delta} \boldsymbol{X}$ & Probability \\
\hline$(1,0,0,0,0,0)$ & $\mu N \Delta t+o(\Delta t)$ & $(0,1,0,-1,0,0)$ & $k_{H} q F_{H} \Delta t+o(\Delta t)$ \\
$(-1,0,0,0,0,0)$ & $\mu V(t) \Delta t+o(\Delta t)$ & $(0,0,-1,0,0,0)$ & $\mu H(t) \Delta t+o(\Delta t)$ \\
$(-1,1,0,0,0,0)$ & $\sigma V(t) \Delta t+o(\Delta t)$ & $(0,0,-1,1,0,0)$ & $\gamma_{H} H(t) \Delta t+o(\Delta t)$ \\
$(1,0,0,0,-1,0)$ & $k_{D} s F_{D} \Delta t+o(\Delta t)$ & $(0,0,1,-1,0,0)$ & $k_{H}(1-q) F_{H}(t) \Delta t+o(\Delta t)$ \\
$(0,-1,1,0,0,0)$ & $\left(\beta_{p} p(t)+\beta_{H} \frac{H(t)}{N}\right) D(t) \Delta t+o(\Delta t)$ & $(0,0,0,-1,0,0)$ & $\mu F_{H}(t) \Delta t+o(\Delta t)$ \\
$(0,-1,0,0,0,0)$ & $\mu D(t) \Delta t+o(\Delta t)$ & $(0,0,0,0,-1,0)$ & $\mu F_{D}(t) \Delta t+o(\Delta t)$ \\
$(0,-1,0,0,1,0)$ & $\gamma_{D} D(t) \Delta t+o(\Delta t)$ & $(0,0,0,0,0,1)$ & $\frac{H(t)}{N(t)} p(t)\left(N_{p}-p(t)\right) \Delta t+o(\Delta t)$ \\
$(0,1,0,0,-1,0)$ & $k_{D}(1-s) F_{D}(t)+o(\Delta t)$ & $(0,0,0,0,0,-1)$ & $\epsilon p(t) \Delta t+o(\Delta t)$ \\
\hline
\end{tabular}

Table 2: Possible state changes $\Delta X=\left(\Delta V, \Delta D, \Delta H, \Delta F_{H}, \Delta F_{D}, \Delta p\right)$ during a small time interval $\Delta t$ : Because the state variable $p \in(0,1)$ is continuous variable, we discretized its domain into $N_{p}$ partitions such that $p(t)$ of them are occupied and $N_{p}-p(t)$ are unoccupied at time $t$.

\section{Analysis}

We now present two main mathematical results of our model, and provide the mathematical derivation details in Appendix.

Remark 1 (Equilibria and their Existence and Stability Condition). The model (1) has three real equilibria, including two boundary equilibria and one interior equilibrium. First we Define

$$
L_{H}:=\left(\mu+\gamma_{H}\right)\left(\mu+\kappa_{H}\right), \quad L_{D}:=\left(\mu+\gamma_{D}\right)\left(\mu+\kappa_{D}\right),
$$

and

$$
M_{H}:=\kappa_{H} \gamma_{H}, \quad M_{D}:=\kappa_{D} \gamma_{D}, \quad K:=\mu \beta_{H}\left(\mu+\kappa_{H}\right)\left(\mu+\kappa_{D}\right)
$$

then we have following conditions for existence of boundary equilibria:

- The first boundary equilibrium Homeless-free HFE (when $H=0$ and $p=0$ ) always exists and is stable if the threshold condition $\mathcal{R}_{H D}$ in Remark 2 is less than one.

- The second boundary equilibrium Environment-free EFE (when only $p=0$ ) exist if

$$
\frac{\left[\left(L_{D}-M_{D}\right)+\frac{\mu}{\sigma}\left(L_{D}-(1-s) M_{D}\right)\right]\left[\left(L_{H}-(1-q) M_{H}\right)\right]}{K}>1 .
$$

Finding explicit formula for stability condition for EFE was mathematically cumbersome, but we investigated it numerically and found out it is unstable (see Result Section). 
- The interior equilibrium EE can be calculated by finding root a cubic polynomial (see Appendix) and it is feasible if

$$
\begin{gathered}
\frac{\mu^{2}+\left(\gamma_{H}-\beta_{p} \kappa_{H}\right) \mu}{\beta_{p} \kappa_{H}}<1, \\
{\left[\sigma+(1-s) M_{D} \mu-(\sigma+\mu)\left(\left(\kappa_{D}+\mu\right) \beta_{p}(1-\epsilon)+L_{D}\right)\right] \times\left[\frac{L_{H}-(1-q) M_{H}}{\kappa_{H}+\mu}-\beta_{p}\right]>0}
\end{gathered}
$$

and

$$
\epsilon N<H^{*} .
$$

The first two condition guarantee that $H^{*}$ and $D^{*}$ are positive, and the last one is needed to have $p^{*}$ as a positive value. Similar to EFE, finding explicit formula for stability condition for EE was mathematically cumbersome, but we investigated it numerically and found out it is locally stable if if $\mathcal{R}_{H D}>1$ (see Result Section).

Remark 2 (Control Reproduction Number calculated at HFE). We calculated the control reproductive number of the model (1) via next generation approach [33]:

$$
\mathcal{R}_{H D}=\frac{\beta_{H} \sigma\left(k_{H}+\mu\right)\left(k_{D}+\mu\right)}{\left(\mu\left(\gamma_{H}+k_{H}+\mu\right)+\gamma_{H} k_{H} q\right)\left(\left(\gamma_{D}+\mu\right)(\sigma+\mu)+k_{D}\left(\mu+\gamma_{D} s+\sigma\right)\right)} .
$$

$\mathcal{R}_{\mathcal{H D}}$ can be written as

$$
\mathcal{R}_{\mathcal{H D}}=\mathcal{R}_{0} \cdot c_{H} \cdot c_{D}
$$

where $\mathcal{R}_{0}=\frac{\beta_{H} \sigma}{\mu(\sigma+\mu)}$ in the basic reproduction number, reproduction number in the absence of housing assistance $F_{H}$ and job training $F_{D}$. The parameter

$$
c_{H}=\frac{k_{H}+\mu}{k_{H}+\mu+\gamma_{H}+\frac{\gamma_{H} k_{H} q}{\mu}}
$$

represents the proportion of individuals who enter the housing assistance program and successfully obtain permanent housing, and the parameter

$$
c_{D}=\frac{k_{D}+\mu}{k_{D}+\mu+\gamma_{D}+\frac{k_{D} \gamma_{D} s}{\sigma+\mu}}
$$

is the proportion of individuals who enter job training programs and successfully exit them by moving to vulnerable compartment. The $\gamma_{D}$ and $\frac{k_{D} \gamma_{D} s}{\sigma+\mu}$ terms represent a successful cycle through the job training program. Therefore, the two intervention strategies, when incorporated together, both work to decrease the control reproductive number $\mathcal{R}_{H D}$ with their respective mechanisms as already described. 


\section{Result}

The goal of this study is to (i) estimate (for the first time) the poverty alleviation, social, and environmental influence rates for L.A county, California in Subsection 4.1, (ii) use simulations to identify the conditions for eliminating homelessness in the presence and absence various interventions in Subsection 4.2. (iii) conduct sensitivity analysis to understand what parameters and how they effect the likelihood of eliminating homelessness in Subsection 4.3, and (iv) find out the efficiency of the two interventions in eliminating homelessness based on neighborhood economic status in Subsection 4.4. Assuming total number of people is constant, the results are reported as the fraction of people in each state shown by lower case letters. For the all the simulations we use the model baseline parameters in Table (4), unless stated otherwise.

\subsection{Parameter estimation}

In order to analyze the performance of the numerical simulations, we need to provide baseline values for the parameters of the model. Most of the parameters are taken from the literature, while the parameters $\beta_{p}, \beta_{H}$, and $\epsilon$ have to be estimated according to the best fit to the data found. For the data, we found time-series of Vulnerable $(V)$, Disaffiliated $(D)$, Homeless $(H)$, and poverty rate $p$ for California State during $2010-2018$.

The percentage distribution of household income in the United States from 2010 to 2018 was reported in Statista [34. The percentage whose income is less than 15, 000 per year was used to find Vulnerable $V$ population. For Disaffiliated population $D$, we used unemployment rate in California State from 2010 to 2018 reported in FRED [35. For homelessness, USICH provided California homelessness statistics during $2010-2018$ [36]. After finding the values for $V$, $D$ and $H$, we scaled them by finding total population as $N=V+D+H$ and then dividing each value by total population: $v=\frac{V}{N}, d=\frac{D}{N}, h=\frac{H}{N}$. And finally, The U.S. census data reports the poverty rate in California per year [12. Assuming that the high poverty area $p$ grows at the same rate as people fall under the poverty line, and influence of environment is independent from the influence of social interaction with homeless people, we use the information of years 2010-2018 for the state variable $p$. The Table (3) shows all the mentioned data.

Estimating poverty alleviation rate $\boldsymbol{\epsilon}$ : Firstly, we estimate poverty alleviation rate $\epsilon$ using the equation $p^{\prime}(t)=h p(1-p)-\epsilon p$. we assume $\mathrm{h}$ is a constant $\bar{h}$ (as the average of homelessness during $2010-2018$ ) to get equation for $p^{\prime}$ as $p^{\prime} \approx \bar{h} p(1-p)-\epsilon p$ and solve it analytically:

$$
p(t) \approx \frac{p(0)(\bar{h}+1) e^{(\bar{h}-\epsilon) t}}{1+\bar{h} e^{(\bar{h}-\epsilon) t}} .
$$

Then fitting the equation (3) to the time series of the poverty rate in LA, we can estimate $\epsilon$, Figure $3 \mathrm{a}$ ).

Estimating influence rates $\beta_{\boldsymbol{H}}$ and $\beta_{\boldsymbol{p}}$ : To estimate $\beta_{H}$ and $\beta_{p}$, we assume there is no intervention, that is, all the parameters related to job training and 


\begin{tabular}{|c|c|c|c|c|c|c|c|c|c|c|}
\hline & \multicolumn{9}{|c|}{ Year } & \multirow[b]{2}{*}{ Ref. } \\
\hline & 2010 & 2011 & 2012 & 2013 & 2014 & 2015 & 2016 & 2017 & 2018 & \\
\hline $\begin{array}{l}\text { Poverty rate in } \\
\text { California }\end{array}$ & $15.3 \%$ & $15.8 \%$ & $15.1 \%$ & $14.6 \%$ & $15.2 \%$ & $13.3 \%$ & $12.8 \%$ & $11.9 \%$ & $11.2 \%$ & 12 \\
\hline $\begin{array}{l}\text { Vulnerability } \\
\text { rate in Califor- } \\
\text { nia }\end{array}$ & $11.4 \%$ & $11.9 \%$ & $11.6 \%$ & $11.6 \%$ & $11.8 \%$ & $10.8 \%$ & $10.6 \%$ & $10.3 \%$ & $10.2 \%$ & 34 \\
\hline $\begin{array}{l}\text { Unemployment } \\
\text { rate in Califor- } \\
\text { nia }\end{array}$ & $12.2 \%$ & $11.7 \%$ & $10.4 \%$ & $8.9 \%$ & $7.5 \%$ & $6.2 \%$ & $5.5 \%$ & $4.8 \%$ & $4.2 \%$ & 35 \\
\hline $\begin{array}{l}\text { Homelessness } \\
\text { rate in Califor- } \\
\text { nia }\end{array}$ & $19.38 \%$ & $20.06 \%$ & $19.32 \%$ & $20.08 \%$ & $19.77 \%$ & $20.5 \%$ & $21.48 \%$ & $24.28 \%$ & $23.55 \%$ & 36 \\
\hline
\end{tabular}

Table 3: The percentage of state variables of the model corresponding to California state over the last eight years.

housing assistance are zero, then we plug the formula (3) in the revised and scaled version of differential equation corresponding to homeless class:

$$
\frac{d h}{d t} \approx\left(\beta_{p} p+\beta_{H} h\right) \bar{d}-\mu h,
$$

where the constant $\bar{d}$ is the average of d during the $2010-2018$ in L.A. Fitting the solution $h$ of equation (4) to the time series of homelessness rate in L.A., and using this fit and optimization toolboxes in Matlab, we can estimate find $\beta_{p}$ and $\beta_{H}$, Figure (3b). Table (4) lists the found and estimated baseline values

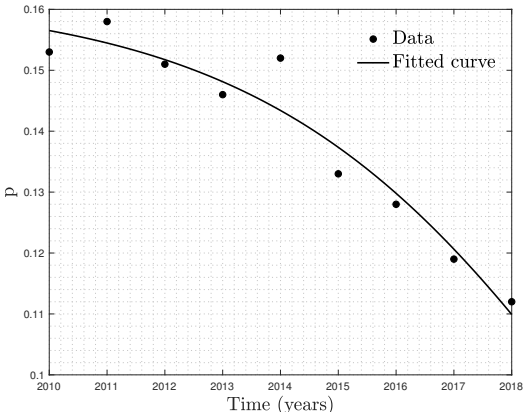

(a) Poverty rate in California in $2010-2018$

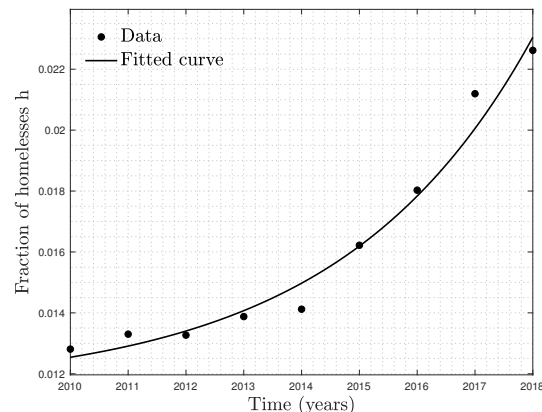

8 (b) Homelessness in California in $2010-$ 2018

Figure 2: Fitted curve to the data of poverty rate and homelessness during the last 8 years in California: The data, solution of model, and fitting and optimization toolboxes in Matlab were used to find unknown parameters poverty alleviation rate $\epsilon$, environmental influence rate $\beta_{p}$ and homeless influence rate $\beta_{H}$.

and range of the parameters of our model. 


\begin{tabular}{clll}
\hline Parameter & Baseline value & Range & Ref. \\
\hline$\sigma$ & 0.25 year $^{-1}$ & $0.2484-0.2516$ & {$[12]$} \\
$\mu$ & 0.013 year $^{-1}$ & $0.012-0.014$ & {$[37]$} \\
$\beta_{p}$ & 0.062 year $^{-1}$ & $95 \%$ CI: $[0.049,0.075]$ & Estimated \\
$\beta_{H}$ & 0.783 year $^{-1}$ & $95 \%$ CI: $[0.373,1.194]$ & Estimated \\
$\epsilon$ & 0.339 year $^{-1}$ & $95 \%$ CI: $[0.27,0.41]$ & Estimated \\
$\gamma_{D}$ & 2 year $^{-1}$ & $1-4.34$ & {$[38$} \\
$\gamma_{H}$ & 1.71 year $^{-1}$ & $1-2.5$ & {$[39]$} \\
$\kappa_{D}$ & 4 year $^{-1}$ & - & {$[40]$} \\
$\kappa_{H}$ & 0.5 year $^{-1}$ & - & {$[16]$} \\
$s$ & 0.56 & - & {$[40]$} \\
$q$ & 0.65 & $0.586-0.710$ & {$[41]$} \\
\hline
\end{tabular}

Table 4: Parameters baseline value and range: The model parameters describing the dynamic of homelessness, as well as poverty area are estimated using data found in 34, 35, 36. 12, but other parameters were obtained from the literature. The ranges for three parameters $\kappa_{H}, \kappa_{D}$ and $s$ were not found in the literature.

\subsection{Impact of intervention strategies on controlling homelessness}

To determine the effectiveness of two intervention strategies in our modelhousing assistance and job training- we compare the time series of fraction of homeless population by turning the interventions on and off. These scenarios are defined as: 1) No intervention, 2) Only job training, 3) Only housing assistance, and 4) Both interventions, which is combination of housing assistance and job training. We performed the simulations using Continuous Time Markov Chain (CTMC) method, with an ensemble of 250 stochastic runs seeding the same initial condition, and with the model baseline parameters in Table (4), unless stated otherwise. For the initial condition we assume $80 \%$ of population is vulnerable while $10 \%$ disaffiliated and the reminding $10 \%$ is homeless. Figure $(3)$ is the time series of homeless fraction and histogram of homeless fraction at quasi-stationary state for these four different scenarios, and Table (5) is the probability of homelessness elimination given different intervention scenarios.

With no intervention, the homelessness reaches about $93 \%$ of the population. These results makes mathematical sense because in our special case with no interventions, individuals cannot leave the homeless class (see the model (1)). Including only job training intervention, we have a marked but slow decrease in the homelessness after 30 years. Job training program merely slows down the rate at which the homeless population increases to reach a quasi-steady state of around $80 \%$. With the inclusion of only housing assistance program, within 10 years, we have a significant $70 \%$ reduction in homelessness to a nonzero quasistationary state of around $3 \%$. When both intervention strategies are included, the homeless population reaches a low prevalence of less than $1 \%$ within 10 years of implementing these programs. Surprisingly, there is a quick initial decrease in homeless prevalence when the temporary housing program is in use.

The major conclusion from this stochastic run is that to treat the home- 


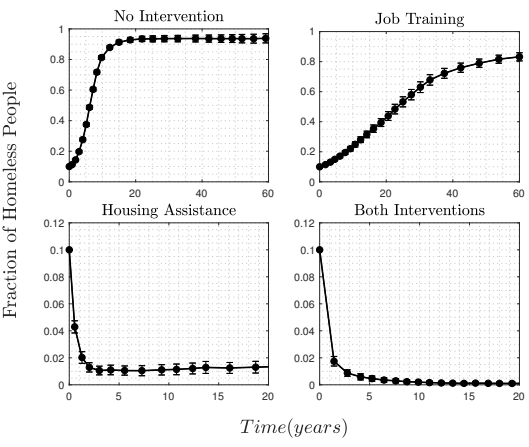

(a) Time series of homelessness

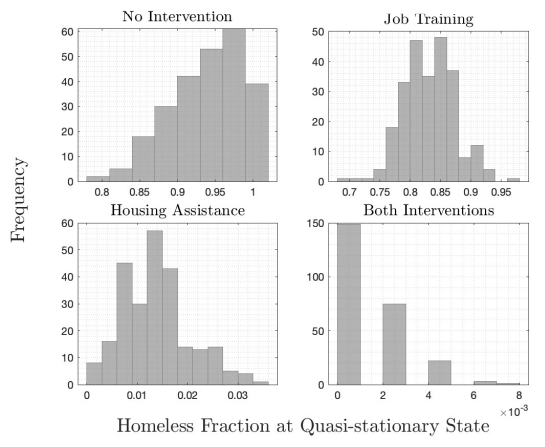

(b) Homeless frequency at quasi-stationary state

Figure 3: Homeless fraction for four different intervention scenarios: (a) The circles are the average of 250 different stochastic simulations, and the error bars are $95 \%$ confidence intervals. For no intervention $\mathcal{R}_{H D}=57.25$ and fraction of homeless people quickly grows to around $90 \%$. when we implement job training $\mathcal{R}_{H D}=9.97$, and in the long run we have around $80 \%$ homelessness. When housing assistance $\mathcal{R}_{H D}=0.65$, and when both interventions implemented $\mathcal{R}_{H D}=0.11$, and for these two scenarios homeless population decays to less than $5 \%$ of population.

lessness issue in the short term, consistent housing assistance is the effective program. But, although not as effective as housing assistance, providing job training in the long term reduces the speed of homelessness growth, therefore, disaffiliated individuals need to be provided with a steady income, and a sustainable approach to prevent risk of being homeless.

\begin{tabular}{ccc}
\hline $\begin{array}{c}\text { Intervention } \\
\text { scenario }\end{array}$ & $\begin{array}{c}\text { Probability of } \\
\text { homelessness elimination }\end{array}$ & $\begin{array}{c}\text { Average fraction of homeless (disaffiliated) } \\
\text { at quasi-stationary state }\end{array}$ \\
\hline No intervention & 0 & $0.966(0.017)$ \\
Job training & 0 & $0.837(0.019)$ \\
Housing assistance & 0.012 & $0.015(0.893)$ \\
Both interventions & 0.596 & $0.00(0.165)$ \\
\hline
\end{tabular}

Table 5: Estimated probability of homelessness elimination in L.A county given current intervention rate and population structure: The calculated probabilities in the second column are the number of zero quasi-stationary states over total number of simulations for given strategy. The fraction of disaffiliated individuals in the third column show how intervention scenarios are affecting disaffiliated subpopulation, for example for no intervention the disaffiliated fraction is less as homeless state is an absorbing state.

\subsection{Global sensitivity analysis}

Sensitivity analysis is defined as how the uncertainty in the output of a mathematical model or system can be divided and allocated to different sources of uncertainty in its parameters 42. In order to determine the role that each parameter plays in the dynamics of the system, it is necessary to conduct sensitivity analysis considering the parameters involved in it. We use Latin Hypercube Sampling to explore the robustness of model results -control reproduction 
number $\mathcal{R}_{H D}$ and fraction of homeless people at stationary state for thew deterministic model (1)- to uncertainty in a 11-dimensional parameter space.

We choose the sample size $n=1000$ to give the partial rank correlation coefficient (PRCC) of $\mathcal{R}_{H D}$ and fraction of homeless individuals at stationary state with respect to our parameter ranges. The empiric distributions of nine parameters in the Latin Hypercube Sampling are represented in Figure (4). The
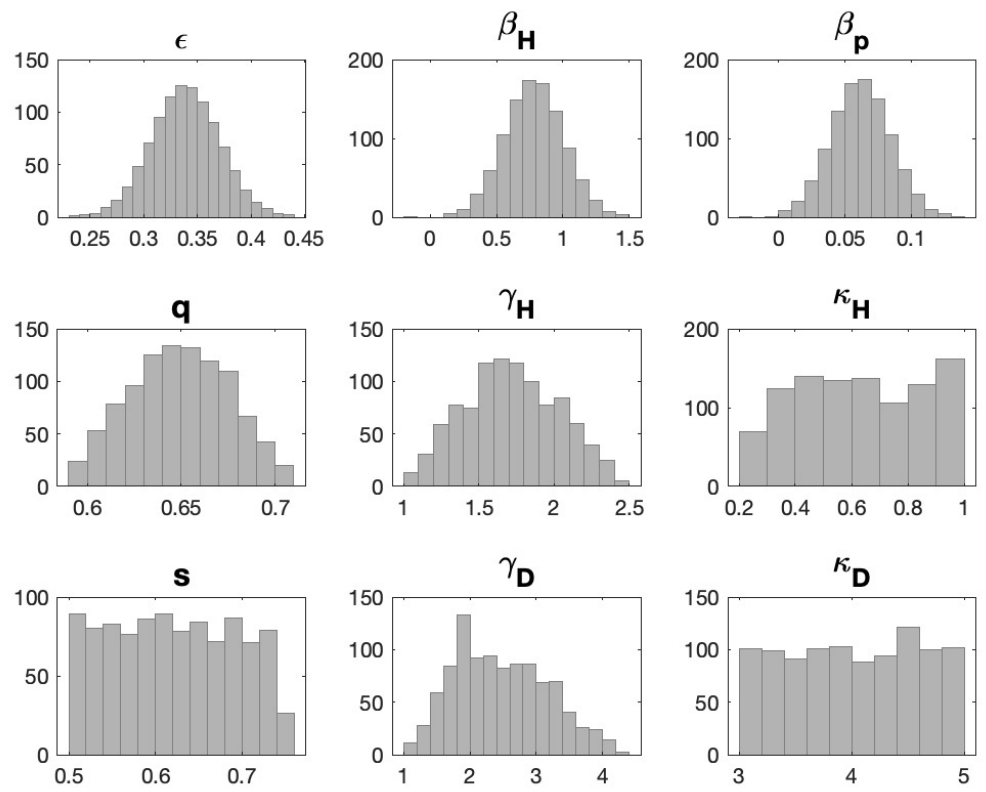

Figure 4: Histograms of the nine parameters in the sample generated using Latin Hypercube Sampling: The parameter distribution for estimated parameters is normal in which negative sample $a$ is substituted by $2 \mu-a$, for parameters with provided range is triangular where the peak is at baseline value, and it is uniform otherwise.

Figure (5) of PRCC reveals us that the parameters related to housing assistance intervention, that is, $q$ and $\gamma_{H}$ are the most effective parameters to reduce $\mathcal{R}_{H D}$ and to control homelessness: increasing housing assistance success $q$ or housing assistance rate $\gamma_{H}$ by $1 \%$ can reduce homelessness by $0.7 \%$ or $0.4 \%$ respectively. On the other hand, homeless influence rate $\beta_{H}$, then followed by environment influence rate $\beta_{p}$, are the most effective parameter to increase homelessness: reducing value for $\beta_{H}$ by $1.1 \%$ or $\beta_{p}$ by $1.7 \%$ decreases the homelessness at steady-state by $1 \%$. Homeless influence rate $\beta_{H}$ is the second most influential parameter to increase $\mathcal{R}_{H D}$. That is, the influence of homeless individuals on the disaffiliated ones is the factor that should be controlled the most in order to control the outbreak of homelessness. 


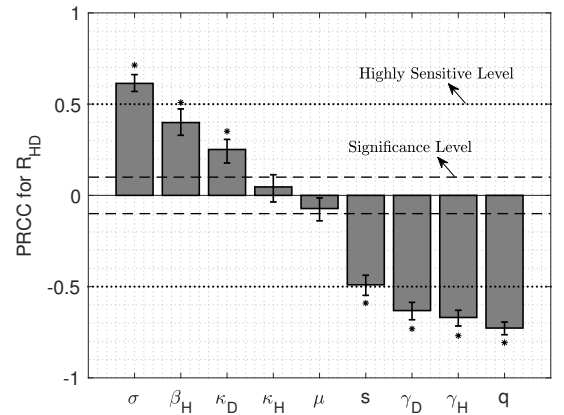

(a) Sensitivity of $\mathcal{R}_{H D}$

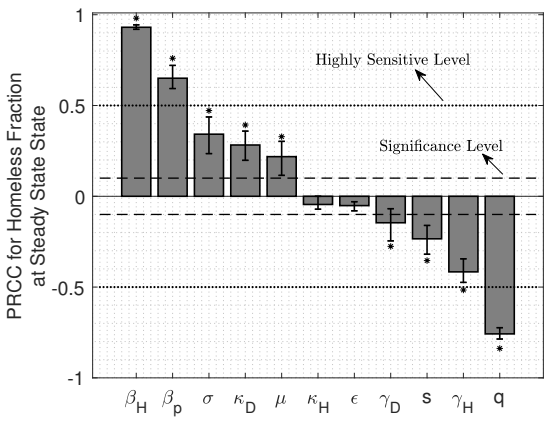

(b) Sensitivity of $H$ at steady state

Figure 5: The partial rank correlation coefficient (PRCC) of the control reproduction number $\mathcal{R}_{H D}$ and homlessness at quasi-stationary state with respect to model parameters: For each parameter 1000 sample is generated in parameter ranges from Latin Hypercube Sampling. The absolute value of PRCC is the sensitivity of the parameterthe larger the value is, the more sensitive outcome is to the corresponding parameter. The notation $*$ denotes the value of PRCC are statistically significant, where the significance level is 0.1 . The most sensitive parameters on homelessness are the influence rate, $\beta_{H}$ and $\beta_{p}$ and parameters related to housing assistance, that is, housing assistance has the most impact in controlling homelessness, while homeless and environment influence rates increase the number of homeless people more effectively than the other parameters.

\subsubsection{Sensitivity of the influence rates on the homelessness}

Because the estimated parameters $\beta_{H}$ and $\beta_{p}$ were the most sensitive parameters to homelessness, and other hand they are most uncertain parameters due to ad hoc method to estimate them, we want to investigate their effectiveness on the homelessness more in depth. We study the behavior of the model (homelessness at steady state) over a confidence interval for these parameters. The Figure (6) displays the results of variations in the homelessness at steady state as function of $\beta_{H}$ and $\beta_{p}$. By definition of $\beta_{H}$ and $\beta_{p}$, qualitatively similar results can be depicted from model (1), increasing the number of homeless people and or impoverished environment can both have important consequences for developing more homeless people. Under increasing $\beta_{H}$ assumptions the number of homeless people always increases nonlinearly; hence, effectiveness was always positive, but under increasing $\beta_{p}$ assumptions the number of homeless people always increases linearly. The Figure $(6)$ also suggests that the influence of an impoverished environment $\beta_{p}$ is less in compare with the influence of social interactions of disaffiliated with homeless individuals $\beta_{H}$ : increasing $\beta_{H}$ over its confidence interval increases homelessness at steady state by $50 \%$, while increasing $\beta_{p}$ over its confidence interval increases homelessness at steady state by $20 \%$. 


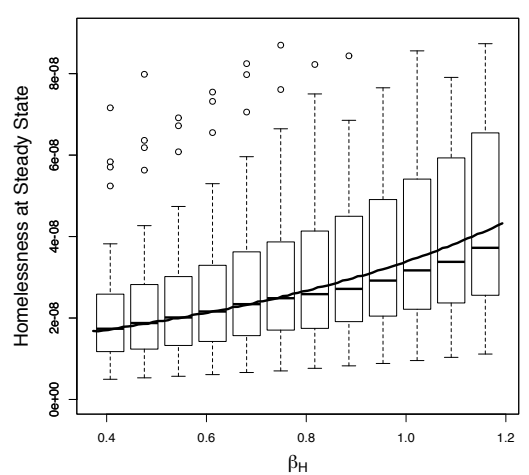

(a) Homeless influence rate

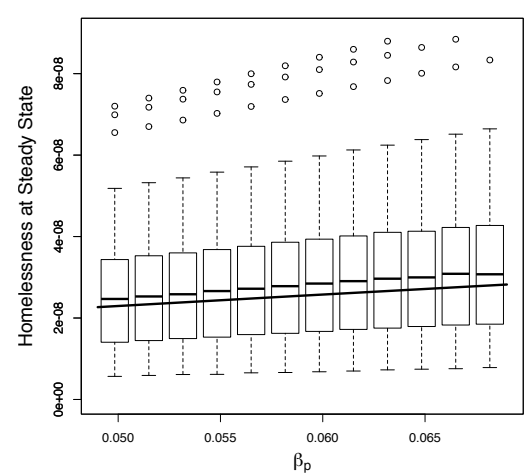

(b) Environment influence rate

Figure 6: Sensitivity analysis showing box plots for the variations in the homelessness at steady state as a function of homeless and environment influence rates, with other parameters sampled from their respective ranges reported in Table (4): The solid curve is the simulation result at baseline values, and each box contains $50 \%$ of data points between the first and third quartiles of the sampling distribution, and whiskers report the remaining $50 \%$ of data points. Increment in homelessness with respect to homeless influence rate $\beta_{H}$ is nonlinear and slightly higher than that of environment influence rate $\beta_{p}$.

\subsubsection{Sensitivity of the intervention strategies on homelessness}

As stated earlier, $s$ and $q$ stand for the proportion of individuals successfully moving from $F_{D}$ to $V$ (job training program was successful to provide them skill sets to find permanent job) and $F_{H}$ to $D$ (Individuals in temporary housing successfully found a permanent house to live) respectively. In order to understand how effective interventions would be on controlling homelessness, we conducted sensitivity analysis on our intervention parameters: the rate of housing assistance implementation $\gamma_{H}$, its long-run success $q$, the rate of job training implementation $\gamma_{D}$, and its long-run success $s$. The Figure (7) shows the variation in homelessness at steady state as a result of changing intervention parameters within their corresponding range in Table (4). For both intervention rates $\gamma_{H}$ and $\gamma_{D}$ we observe qualitatively similar results: For small values of rates, increasing intervention rates decreases homelessness drastically, increasing $\gamma_{H}$ by $35 \%$ decreases homelessness by $85 \%$, and the same increment in $\gamma_{D}$ decreases homelessness by $50 \%$. But, this decrease slows down as the rates becomes bigger, for the rates bigger than 1.5 the plot of homelessness become almost flat, subfigures (7a) and (7c). We also observe that the sensitivity of homelessness to housing assistance rate $\gamma_{H}$ is more than that of job training, as PRCC plot in Figure (5b) was revealing similar result.

For the success of interventions $q$ and $s$ the qualitative results are different: increment in housing assistance success $q$ decreases homelessness via a nonlinear curve meaning that for small values of $q$ change in homelessness is sensitive to 
$q$, but as $q$ increases this sensitivity will decrease, however, the trend between job training success $s$ and homelessness is linear and less sensitive, subfigures (7b) and (7d). These result means that to have housing assistance intervention works for the long run, we need to increase its success beside its rate via some back up plan to make sure sheltered homeless individuals get permanent place to live in.

\subsection{The region-based efficiency of intervention strategies}

In the previous subsections we observed that poverty alleviation rate $\epsilon$ played a negligible impact on the homelessness in L.A., California, Figure (5), which it cab be result of small range for this parameter. Here we aim to understand how changing poverty alleviation rate $\epsilon$ over a wider range can impact the homelessness and efficiency of interventions. We plotted homelessness at steady state versus poverty alleviation rate under the two different scenarios: 1) when job training program is more successful than housing assistance program, that is $s \kappa_{D}=q \kappa_{H}$ and $s>q$ and 2) when housing assistance program is more successful than job training program, that is $s \kappa_{D}=q \kappa_{H}$ and $s<q$. The condition $s \kappa_{D}=q \kappa_{H}$ in both scenarios guarantees that the number of people per time successfully leaving $F_{D}$ and $F_{H}$ is the same, therefore, comparing the interventions is reasonable.

The result plotted in Figure (8) reveals us two interesting facts: First, since both graphs appear to plateau at around $\epsilon=0.3$, we can conclude that the cutoff for the effectiveness of each type of intervention is around $30 \%$ of the designated population, that is, as the intervention successes increase beyond this cutoff value, they exhibit diminishing marginal reductions, with each graph showing horizontal asymptote. Second, if we assume $\epsilon$ is one characteristic of poverty of a region- smaller $\epsilon$ refers to poorer region- then the plot shows that, for poor regions the difference in impact of two programs is almost negligible, however, for the richer regions (higher $\epsilon$ ) the difference between impacts of two programs increases showing that housing assistance program becomes more effective in controlling homelessness. The take home message can be interpreted that in the poor region, the policy makers need to provide more job opportunity to prevent homelessness problem.

\section{Discussion}

We used a compartmental ordinary and stochastic differential equationsbased model combined with Levin's equation 30 to study the impact of environment, homeless density, and two interventions- temporary housing and job training- on the overall homelessness dynamics in L.A. County. We stratified the population at risk of homelessness into three main stages: vulnerable, disaffiliated, and homeless, and implemented the interventions on disaffiliated and homeless subpopulations. We analyzed our model by deriving equilibrium points and their stability conditions and also calculating control reproduction number $\mathcal{R}_{H D}$. There were 11 parameters involved in the model, some of which 


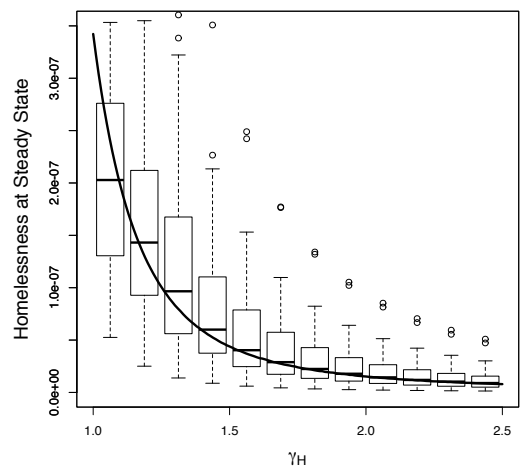

(a) Housing assistance rate

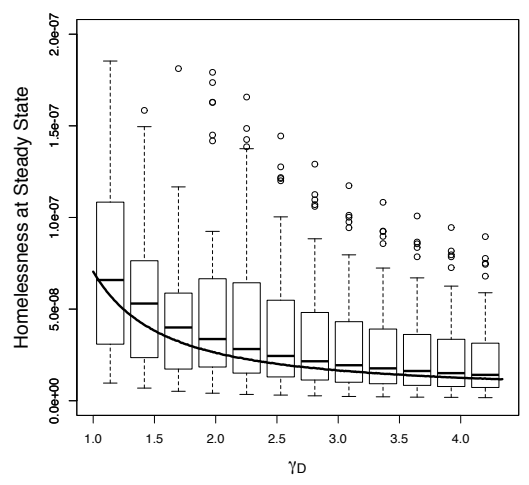

(c) Job training rate

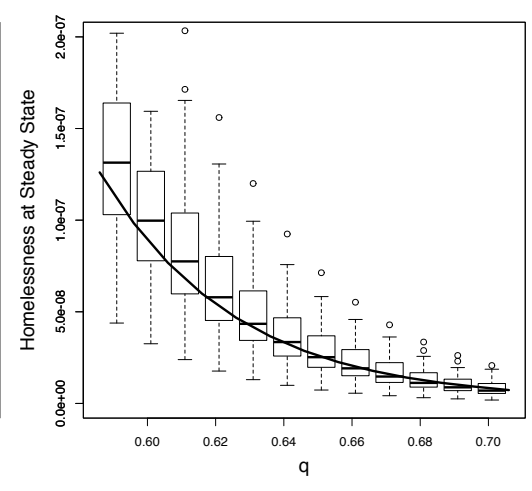

(b) Housing assistance success

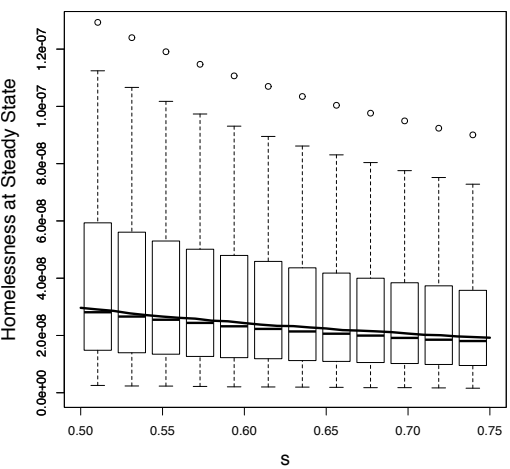

(d) Job training success

Figure 7: Sensitivity analysis showing box plots for the variations in the homelessness at quasi-stationary state as a function of intervention strategies parameters, with other parameters sampled from their respective ranges reported in Table (4): The solid curve is the simulation result at baseline values, and each box contains $50 \%$ of data points between the first and third quartiles of the sampling distribution, and whiskers report the remaining $50 \%$ of data points. Decreasing in homelessness with respect to housing assistance parameters, $\gamma_{H}$ and $q$, is nonlinear and slightly higher than that of job training, $\gamma_{D}$ and $s$. 


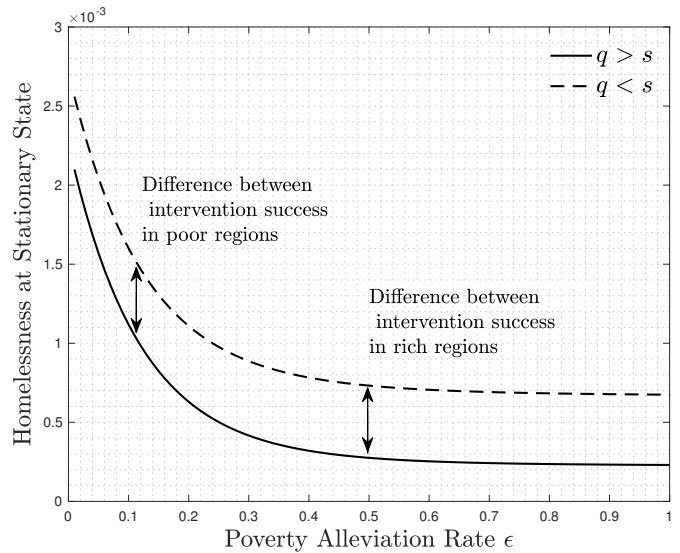

Figure 8: Homelessness at steady state versus $\epsilon$ for two scenarios: When poverty alleviation rate is small, the homelessness decreases drastically as poverty alleviation rate increase, but for bigger values of poverty alleviation rate $(\epsilon>0.3)$ homelessness stabilize to a constant point meaning that removing poverty does not help reduce homelessness. Also poorer regions indicated by small $\epsilon$ are healed from homelessness through providing more job opportunities for disaffiliated people, while this idea is enough to control homelessness in richer regions, regions with higher $\epsilon$.

were found in the literature, the rest were estimated according to the best fit to the time-series data of homelessness in California state during 2010 - 2018. An stochastic continuous time Markov Chain was used to simulate the model, and deterministic model was used to conduct the global sensitivity analysis of the parameters. In order to investigate the sensitivity analysis of the equilibrium points and control reproduction number, we used Latin Hypercube Sampling with sample size of 1000 for each parameter and reported Partial Rank Correlation Coefficient (PRCC) for all involved parameters and box plot of variation of homelessness with respect to most influential parameters.

Our findings suggest that for the short-term reduction on homelessness in L.A. county, the focus of effort should be primarily on temporary housing programs, but that job training is necessary for sustainable reduction of homelessness in the long run. Although job training programs lead to additional reduction in the homelessness, they pale in compare with the benefits that temporary housing brings. Housing assistance programs, as mentioned by Wodon et al. [23, are short-term investment, that is, providing housing will remove homeless individuals from the streets, but only after providing disaffiliated individuals with a source of income the problem be best treated in the long term, Figure (3).

Conducting global sensitivity analysis, we found that homeless and environment influences are the most influential parameters for homelessness at steady state: in order to achieve $1 \%$ decrease in homelessness it is necessary to decrease the value for $\beta_{H}$ by $1.1 \%$ or $\beta_{p}$ by $1.7 \%$. On the other hand, housing 
assistance rate and success $\gamma_{H}$ and $q$ are highly correlated with homelessness at steady state. If it become successful (related to parameter $q$ ) enough housing assistance effort (related to parameter $\gamma_{H}$ ) is the most influential approach to control the number of homeless people at quasi-stationary state: Increasing housing assistance success $q$ or housing assistance rate $\gamma_{H}$ by $1 \%$ can reduce homelessness by $0.7 \%$ or $0.4 \%$ respectively. Lacey et al. 24 found similar result on the rate of housing assistance: the rate at which the homeless are rehoused in council property plays the key factor in size of homeless population and other categories in their model 24. Moderate correlation exists between the $\sigma, \kappa_{D}$, $s, \gamma_{D}$ and homelessness at quasi-stationary state. Weak correlation has been observed between $\kappa_{H}, \epsilon$ and homelessness at quasi-stationary state. For control reproduction number $\mathcal{R}_{H D}$ similar result have been observed, Figure (5).

The poverty alleviation rate $\epsilon$ can be defined as an indicator of poverty of a region: smaller $\epsilon$, poorer region. With that definition we could test efficiency of intervention programs based on poorness of a region. The homelessness is sensitive to both interventions for poorer regions, regions with $\epsilon \leq 0.3$, but, increasing $\epsilon$ shows that more effort of programs would not decrease homelessness to the lower level. Also, as the difference in impact of two programs is almost negligible for small $\epsilon$ values, for poorer regions the focus should be on providing job skill and job opportunity for disaffiliated population, while for rich regions housing assistance program should be the prior program to control homelessness, Figure (8).

Our model, like any other dynamical modelings, has a luxury of experimenting with social issue in a virtual environment which is encapsulated from real world [43], as it can not fully replicate the mirco-level interactions that may be necessary in determining homelessness dynamic. For example, by basing our focus on poverty as the only reason of homelessness, we did not capture other possible reasons such as mental disorder or drug misuse, as they can be strong predictor of homelessness in U.S. [14, 25]. Further, to have a more realistic representation of the system, analyzing the dynamics of the system under a limited budget scenario for the interventions needs to be done, that is, we should look for the optimal investment forwarded to each strategy that makes for the most effective combination. Finally, in order to keep track of how effective the interventions are over time at large time scales, we need to make the transitions between stages dependent on time based on seasonal fluctuations of homelessness [4], to better identify the most optimal time to strengthen each of the interventions. Our preliminary studies indicate that the qualitative findings of this paper are relatively insensitive to adding these additional constraints. Although the model is still too simplest in directly guiding policy makers to alleviate this social issue, the qualitative trends predicted by our simulations can be useful in designing studies to quantify the effectiveness of these two mentioned interventions. 


\section{Acknowledgement}

We thank Dr. José Flores, Dr.Carlos M. Hernández-Suárez, Dr. Christopher Kribs, and anonymous reviewers for their comments on the manuscript. This research was conducted as part of 2019 MTBI at the Simon A. Levin Mathematical, Computational and Modeling Sciences Center (MCMSC) at Arizona State University (ASU). This project has been partially supported by grants from the National Science Foundation (NSF Grant MPS-DMS-1263374 and NSF Grant DMS-1757968), the National Security Agency (NSA Grant H98230-J8-1-0005), the Office of the President of ASU, and the Office of the Provost of ASU.

[1] Thompson Jr, Ronald G and Wall, Melanie M and Greenstein, Eliana and Grant, Bridget F and Hasin, Deborah S. Substance-use disorders and poverty as prospective predictors of first-time homelessness in the United States. American Journal of Public Health, 103(S2):S282-S288, 2013. https://doi.org/10.2105/AJPH.2013.301302.

[2] McChesney, Kay Young. Characteristics of the residents of two inner-city emergency shelters for the homeless. Los Angeles, Calif: University of Southern California Social Science Research Institute, 1987.

[3] Bassuk, Ellen L and Rosenberg, Lynn. Why does family homelessness occur? A case-control study. American Journal of Public Health, 78(7):783-788, 1988.

https://doi.org/10.2105/AJPH.78.7.783

[4] Sosin, Michael R and others. Homelessness in Chicago: Poverty and Pathology, Social Institutions and Social Change. ERIC, 1988.

[5] Shinn, Marybeth and Weitzman, Beth C. Research on homelessness: An introduction. Journal of Social Issues, 46(4):1-11, 1990.

https://doi.org/10.1111/j.1540-4560.1990.tb01795.x.

[6] Greenberg, Greg A and Rosenheck, Robert A. Mental health correlates of past homelessness in the National Comorbidity Study Replication. Journal of health care for the poor and underserved, 21(4):1234-1249, 2010. https://muse.jhu.edu/article/400765\#.XhPbTm_MV_I.gmail.

[7] Charles Grigsby, Donald Baumann, Steven E Gregorich, and Cynthia Roberts-Gray. Disaffiliation to entrenchment: A model for understanding homelessness. Journal of Social Issues, 46(4):141-156, 1990. https://doi.org/10.1111/j.1540-4560.1990.tb01803.x

[8] Hagen, Jan L. The heterogeneity of homelessness. Social Casework, 1987.

[9] Burt, Martha R and Aron, Laudan Y and Lee, Edgar. Helping America's homeless: Emergency shelter or affordable housing? Journal of health care for the poor and underserved, 2001. 
[10] Elijah Chilland. Low-income requirements for Los Angeles updated for 2017.

[11] Meghan Henry, Anna Mahathey, Tyler Morrill, Anna Robinson, Azim Shivji, and Rian Watt. The 2018 annual homeless assessment report (ahar) to congress, 2018.

[12] U.S. Census Bureau. Los angeles county quickfacts. https://www. census.gov/quickfacts/fact/table/\losangelescountycalifornia/ PST045218. Accessed: 2019-07-10.

[13] U.S. Census Bureau. Income and poverty in the united states. https:// www . census.gov/library/publications/2018/demo/p60-263.html. Accessed: 2019-07-10.

[14] Marta Elliott and Lauren J. Krivo. Structural determinants of homelessness in the united states. Social Problems, 38(1):113-131, February 1991. https://doi.org/10.2307/800641.

[15] LA:RISE the Los Angeles Regional Initiative for Social Enterprise, REDF. https://redf workshop.org/larise/what. Accessed: 2019-07-11.

[16] U.S. Department of Housing and Urban Development. Continuum of care program eligibility requirements. https://www.hudexchange.info/ programs/coc/coc-program-eligibility-requirements/. Accessed: 2019-07-10.

[17] Link, Bruce G and Susser, Ezra and Stueve, Ann and Phelan, Joe and Moore, Robert E and Struening, Elmer. Lifetime and five-year prevalence of homelessness in the United States. American Journal of Public Health, 84(12):1907-1912, 1994. https://doi.org/10.2105/AJPH.84.12.1907.

[18] Song, John and Ratner, Edward R and Wall, Melanie M and Bartels, Dianne $\mathrm{M}$ and Ulvestad, Nancy and Petroskas, Dawn and West, Melissa and Weber-Main, Anne Marie and Grengs, Leah and Gelberg, Lillian. Effect of an end-of-life planning intervention on the completion of advance directives in homeless persons: a randomized trial. Annals of internal medicine, 153(2):76-84, 2010.

[19] de Vet, Renee and Beijersbergen, Marielle D and Jonker, Irene E and Lako, Danielle AM and van Hemert, Albert M and Herman, Daniel B and Wolf, Judith RLM. Critical time intervention for homeless people making the transition to community living: a randomized controlled trial. American journal of community psychology, 60(1-2):175-186, 2017. https://doi.org/10.1002/ajcp.12150.

[20] Orwin, Robert G and Scott, Chris K and Arieira, Carlos. Transitions through homelessness and factors that predict them: three-year treatment outcomes. Journal of Substance Abuse Treatment, 28(2):S23-S39, 2005. 
https://doi.org/10.1016/j.jsat.2004.10.011.

[21] Caton, Carol LM and Dominguez, Boanerges and Schanzer, Bella and Hasin, Deborah S and Shrout, Patrick E and Felix, Alan and McQuistion, Hunter and Opler, Lewis A and Hsu, Eustace. Risk factors for long-term homelessness: Findings from a longitudinal study of first-time homeless single adults. American journal of public health, 95(10):1753-1759, 2005. https://doi.org/10.2105/AJPH.2005.063321.

[22] Vincent A Fusaro, Helen G Levy, and H Luke Shaefer. Racial and ethnic disparities in the lifetime prevalence of homelessness in the United States. Demography, 55(6):2119-2128, 2018. https://doi.org/10.1007/s13524-018-0717-0.

[23] Quentin T Wodon. Providing shelters or low income housing? an optimal control model. Journal of Housing Economics, 8(2):90-115, 1999. https://doi.org/10.1006/jhec.1999.0245

[24] AA Lacey, DF Parker, K Parrott, D Simpson, Warren Smith, and JAD Wattis. Modelling of homeless populations.

[25] Bhunu, Claver P. Homelessness and drug misuse in developing countries: A mathematical approach Communications in Nonlinear Science and $\mathrm{Nu}$ merical Simulation, 19(6):1908-1917, 2014. https://doi.org/10.1016/j.cnsns.2013.10.013.

[26] Toro, Paul A and Trickett, Edison J and Wall, David D and Salem, Deborah A. Homelessness in the United States: An ecological perspective. American Psychologist, 46(11):1208, 1991. https://doi.org/10.1037/0003-066X.46.11.1208

[27] Jones, Brian T. The social ecology of homelessness: exploring the dynamics of engagement among homeless street adults. Journal of Human Behavior in the Social Environment, 23(1):53-74, 2013. https://doi.org/10.1080/10911359.2012.739532

[28] Temporary assistance for needy families. https://www.benefits.gov/ benefit/627. Accessed: 2019-07-11.

[29] U.S. Department of Health \& Human Services. 2019 poverty guidelines. https://aspe.hhs.gov/2019-poverty-guidelines. Accessed: 2019-0709 .

[30] Richard Levins. Some demographic and genetic consequences of environmental heterogeneity for biological control. American Entomologist, 15(3):237-240, 1969. https://doi.org/10.1093/besa/15.3.237. 
[31] Takefumi Nakazawa. Introducing stage-specific spatial distribution into the Levins metapopulation model. Scientific reports, 5:7871, 2015. https://doi.org/10.1038/srep07871.

[32] Sven Erik Jørgensen and Brian D. Fath. Developments in Environmental Modelling, 23, 347-368, 2011. https://doi.org/10.1016/B978-0-444-53567-2.00011-9.

[33] Odo Diekmann, John Heesterbeek, Peter Andre, and Johan AJ Metz. On the definition and the computation of the basic reproduction ratio $R_{0}$ in models for infectious diseases in heterogeneous populations. Journal of mathematical biology, 28(4):365-382, 1990. https://doi.org/10.1007/BF00178324.

[34] Statista. Household income in the U.S. - percentage distribution 2006-2017 https://www.statista.com/statistics/758502/ percentage-distribution-of-household-income-in-the-us/.

[35] FRED Unemployment Rate in California https://fred.stlouisfed. org/series/CAUR.

[36] United States Interagency Council of Homelessness California Homelessness Statistics https://www.usich.gov/homelessness-statistics/ca.

[37] National Vital Statistics Reports. United states life tables, 2017. https: //www.cdc.gov/nchs/data/nvsr/nvsr68/nvsr68_07-508.eps.

[38] Lisa Guerin. Amount and duration of unemployment compensation in California. https://www.nolo.com/legal-encyclopedia/ collecting-unemployment-benefits-california-32504-2.html.

[39] The National Coalition for the Homeless. Why are people homeless? https://www .nationalhomeless.org/factsheets/why.html.

[40] Dana Rotz, Nan Maxwell, Adam Dunn, et al. Economic self-sufficiency and life stability one year after starting a social enterprise job. 2015.

[41] U.S. Department of Housing and Urban Development. Homeless prevention and rapid re-housing program year 2 summary. 2013.

[42] Andrea Saltelli. Sensitivity analysis for importance assessment. Risk Analysis, 22(3):579-590, June 2002. https://doi.org/10.1111/0272-4332.00040.

[43] Morden, Hilary K and Fritz, Charles and Wu, Tiankuang and Namazi, Sara and Geranmayeh, Parastoo and Chattopadhyay, Rakhi. Analyzing the Impact of Social Factors on Homelessness: A Fuzzy Cognitive Map Approach. BMC medical informatics and decision making, 13(1):94, 2013.

https://doi.org/10.1186/1472-6947-13-94. 
[44] Lee, Barrett A. Stability and change in an urban homeless population. Demography, 26(2):323-334, 1989. https://doi.org/10.2307/2061529.

\section{Appendix}

\subsection{Equilibra of the model}

Putting right hand side of the model (1) equal to zero and solving for the state variable, we find Equlibria as below:

1. HFE: or Homeless Free Equilibrium point is the one for which $H^{*}=p^{*}=$ 0 . For this case we have

- $D^{*}=\frac{\sigma \mu N}{(\sigma+\mu)\left(\gamma_{D}+\mu\right)\left(1-\frac{(\sigma+(1-s) \mu) \kappa_{D} \gamma_{D}}{(\sigma+\mu)\left(\kappa_{D}+\mu\right)\left(\gamma_{D}+\mu\right)}\right)}$

- $V^{*}=\frac{\mu N}{\sigma+\mu}+\frac{s \kappa_{D} \gamma_{D} D^{*}}{(\sigma+\mu)\left(\kappa_{D}+\mu\right)}$

- $H^{*}=0$

- $F_{H}^{*}=0$

- $F_{D}^{*}=\frac{\gamma_{D} D^{*}}{\kappa_{D}+\mu}$

- $p^{*}=0$

2. EFE: or Environment Free Equilibrium point is the one for which $p^{*}=0$. For this case we have

- $D^{*}=\frac{N\left(\kappa_{H}+\mu\right)\left(\gamma_{H}+\mu\right)-(1-q) N \kappa_{H} \gamma_{H}}{\beta_{H}\left(\kappa_{H}+\mu\right)}$

- $V^{*}=\frac{\mu N}{\sigma+\mu}+\frac{s \kappa_{D} \gamma_{D} D^{*}}{(\sigma+\mu)\left(\kappa_{D}+\mu\right)}$

- $F_{D}^{*}=\frac{\gamma_{D} D^{*}}{\kappa_{D}+\mu}$

- $H^{*}=\frac{\left(\gamma_{D}+\mu\right) D^{*}-(1-s) \kappa_{D} F_{D}^{*}-\sigma V^{*}}{\frac{q \kappa_{H} \gamma_{H}}{\kappa_{H}+\mu}-\frac{\beta_{H} D^{*}}{N}}$

- $F_{H}^{*}=\frac{\gamma_{H} H^{*}}{\kappa_{H}+\mu}$

- $p^{*}=0$

3. EE: or endemic equilibria for which all the state variable are non-zero.

- $D^{*}=\frac{\left(\kappa_{H}+\mu\right) \sigma \mu N+q(\sigma+\mu) \kappa_{H} \gamma_{H} H^{*}}{\left[\beta_{H} \frac{H^{*}}{N}+\beta_{p}\left(1-\epsilon \frac{N}{H^{*}}\right)+\mu+\gamma_{D}\right]\left[1-\frac{(\sigma+(1-s) \mu) \kappa_{D} \gamma_{D}}{\left(\kappa_{D}+\mu\right)(\sigma+\mu)\left[\beta_{H} \frac{H^{*}}{N}+\beta_{p}\left(1-\epsilon \frac{N}{H^{*}}\right)+\mu+\gamma_{D}\right]}\right](\sigma+\mu)\left(\kappa_{H}+\mu\right)}$.

- $V^{*}=\frac{\mu N}{\sigma+\mu}+\frac{s \kappa_{D} \gamma_{D} D^{*}}{(\sigma+\mu)\left(\kappa_{D}+\mu\right)}$.

- $F_{D}^{*}=\frac{\gamma_{D} D^{*}}{\kappa_{D}+\mu}$

- $F_{H}^{*}=\frac{\gamma_{H} H^{*}}{\kappa_{H}+\mu}$

- $p^{*}=1-\frac{\epsilon N}{H^{*}}$ 
where, $H^{*}$ is the positive root of the cubic polynomial $a_{3} x^{3}+a_{2} x^{2}+a_{1} x+a_{0}$ where

$$
\begin{aligned}
& a_{3}=-N \beta_{H}\left(\kappa_{D}+\mu\right)(\sigma+\mu)^{2}\left[\mu^{2}+\left(\gamma_{H}-\beta_{p} \kappa_{H}\right) \mu-\beta_{p} \kappa_{H}\right], \\
& a_{2}=N^{2}\left(\left[\sigma+(1-s) \gamma_{D} \kappa_{D} \mu-\left(\kappa_{D}+\mu\right)(\sigma+\mu)\left(\beta_{p}(1-\epsilon)+\gamma_{D}+\mu\right)\right] \times\left[\gamma_{H}+\mu-\frac{(1-q) \gamma_{H} \kappa_{H}}{\kappa_{H}+\mu}\right]\right. \\
& \left.+\left[\left(\kappa_{D}+\mu\right)(\mu+\sigma)\left((1-\epsilon) \beta_{p}+\gamma_{D}+\mu\right)-\sigma-(1-s) \gamma_{D} \kappa_{D} \mu\right)\right] \beta_{p} \\
& \left.+\mu \sigma\left(\kappa_{D}+\mu\right)\left(\kappa_{H}+\mu\right)(\sigma+\mu)\right), \\
& \quad \\
& a_{1}=N^{3} \beta_{p} \epsilon \gamma_{H} \kappa_{H} q\left(\kappa_{D}+\mu\right)(\sigma+\mu)^{2}, \quad \text { and, } a_{0}=-N^{4} \beta_{p} \epsilon \mu \sigma\left(\kappa_{D}+\mu\right)\left(\kappa_{H}+\mu\right)(\sigma+\mu) .
\end{aligned}
$$

Because $a_{0} \neq 0$ this polynomial has exactly one real positive root if $a_{3}>0$ and $a_{2}>0$, Descartes Sign Rule.

\subsection{Control reproduction number}

The control reproduction number was obtained through the next generation approach 33. at HFE. First, we established the matrices $\mathcal{F}$ and $\mathcal{V}$ with components of direction transmission and other transition processes, respectively; obtaining the following:

$$
\mathcal{F}=\left(\begin{array}{c}
\beta(H, p) D \\
0 \\
\Omega(H) p(1-p)
\end{array}\right), \quad \mathcal{V}=\left(\begin{array}{c}
\left(\mu+\gamma_{H}\right) H-\kappa_{H}(1-q) F_{H} \\
-\gamma_{H} H+\left(\kappa_{H}+\mu\right) F_{H} \\
\varepsilon p
\end{array}\right)
$$

Jacobians of the above expression provide the following matrices:

$$
F=\left(\begin{array}{ccc}
\beta_{H} \frac{D}{N} & 0 & \beta_{p} D \\
0 & 0 & 0 \\
\frac{1}{N} p(1-p) & 0 & \frac{H}{N}(1-2 p)
\end{array}\right) \quad V=\left(\begin{array}{ccc}
\gamma_{H}+\mu & -(1-q) \kappa_{H} & 0 \\
-\gamma_{H} & \kappa_{H}+\mu & 0 \\
0 & 0 & \varepsilon
\end{array}\right)
$$

Now, substituting the HFE into $F$ and $V$ provides the following results:

$$
\begin{gathered}
F_{H F E}=\left(\begin{array}{ccc}
\frac{\beta_{H} \sigma\left(k_{D}+\mu\right)}{(\mu+\sigma)\left(\mu+\kappa_{D}\right)+\gamma_{D}\left(\mu+\sigma+s \kappa_{D}\right)} & 0 & \frac{\beta_{p} \sigma\left(k_{D}+\mu\right)}{(\mu+\sigma)\left(\mu+\kappa_{D}\right)+\gamma_{D}\left(\mu+\sigma+s \kappa_{D}\right)} \\
0 & 0 & 0 \\
0 & 0 & 0
\end{array}\right) \\
V_{H F E}=\left(\begin{array}{ccc}
\gamma_{H}+\mu & (q-1) \kappa_{H} & 0 \\
-\gamma_{H} & \kappa_{H}+\mu & 0 \\
0 & 0 & \varepsilon
\end{array}\right)
\end{gathered}
$$

Multiply $F V^{-1}$ and find the largest eigenvalue to obtain the reproductive number:

$$
\begin{aligned}
\mathcal{R}_{H D} & =\frac{\beta_{H} \sigma\left(k_{H}+\mu\right)\left(k_{D}+\mu\right)}{\left(\mu\left(\gamma_{H}+k_{H}+\mu\right)+\gamma_{H} k_{H} q\right)\left(\left(\gamma_{D}+\mu\right)(\sigma+\mu)+k_{D}\left(\mu+\gamma_{D} s+\sigma\right)\right)} \\
& =\frac{\beta_{H} \sigma}{\mu(\sigma+\mu)} \times \frac{k_{H}+\mu}{k_{H}+\mu+\gamma_{H}+\frac{\gamma_{H} k_{H} q}{\mu}} \times \frac{k_{D}+\mu}{k_{D}+\mu+\gamma_{D}+\frac{k_{D} \gamma_{D} s}{\sigma+\mu}} .
\end{aligned}
$$

